\title{
2-Benzoyl-6-benzylidenecyclohexanone analogs as potent dual inhibitors of acetylcholinesterase and butyrylcholinesterase
}

\begin{abstract}
In the present study, a series of 2-benzoyl-6-benzylidenecyclohexanone analogs have been synthesized and evaluated for their anti-cholinesterase activity. Among the forty-one analogs, four compounds $(\mathbf{3 8}, \mathbf{3 9}, \mathbf{4 0}$ and $\mathbf{4 1})$ have been identified as lead compounds due to their highest inhibition on both $\mathrm{AChE}$ and $\mathrm{BChE}$ activities. Compounds 39 and $\mathbf{4 0}$ in particular exhibited highest inhibition on both $\mathrm{AChE}$ and $\mathrm{BChE}$ with $\mathrm{IC}_{50}$ values of $1.6 \mu \mathrm{M}$ and $0.6 \mu \mathrm{M}$, respectively. Further structure-activity relationship study suggested that presence of a longchain heterocyclic in one of the rings played a critical role in the dual enzymes' inhibition. The Lineweaver-Burk plots and docking results suggest that both compounds could simultaneously bind to the PAS and CAS regions of the enzyme. ADMET analysis further confirmed the therapeutic potential of both compounds based upon their high BBBpenetrating. Thus, 2-benzoyl-6-benzylidenecyclohexanone containing long-chain heterocyclic amine analogs represent a new class of cholinesterase inhibitor, which deserve further investigation for their development into therapeutic agents for cognitive diseases such as Alzheimer.
\end{abstract}

Keyword: Acetylcholinesterase; Butyrylcholinesterase; 2-Benzoyl-6benzylidenecyclohexanone; Kinetic studies; Molecular docking 